CP, 2014, N5, pp. 74-90. ISSN 2014-6752. Girona (Catalunya). SANTOS, Anabela; CABECINHAS, Rosa y CERQUEIRA, Carla: Leer, Interpretar y (Re)construir: Percepciones de Jóvenes sobre las Mujeres en la Política.

Reading, Interpreting and (Re)constructing: Youth Perceptions about Women in Politics.

Recibido: 18/12/2014 - Aceptado: 22/12/2014

\title{
LEER, INTERPRETAR Y (RE)CONSTRUIR: PERCEPCIONES DE JÓVENES SOBRE LAS MUJERES EN LA POLÍTICA
}

\section{Reading, Interpreting and (Re)constructing: Youth Perceptions about Women in Politics}

\section{Autoras: SANTOS, Anabela; CABECINHAS, Rosa y CERQUEIRA, Carla}

Becaria de Inverstigación - Universidade do Minho - Portugal-amsantos86@gmail.com

Profesora Asociada - Universidade do Minho - Portugal-cabecinhas@ics.uminho.pt

Investigadora de Pós-Doctorado (Universidade do Minho) y Profesora Auxiliar (Universidade Lusófona do

Porto) - Portugal - carlaprec3@gmail.com

Centro de Estudos de Comunicação e Sociedade - Instituto de Ciências Sociais - Universidade do Minho

\section{Resumen}

El presente artículo analiza las prácticas de recepción de contenidos difundidos por las revistas portuguesas de información generalista "Sábado" y "Visão", estudiando, en concreto, el modo en el que los públicos jóvenes comprenden, interpretan y se sitúan en relación a las representaciones de mujeres que ejercen cargos políticos.

El análisis temático conducido, el cual partió del material resultante de la realización de once grupos focales, muestra que las/los participantes tendieron a favorecer lecturas congruentes con la ideología tradicional de género. Independientemente de los momentos puntuales de negociación, controversia y resistencia, los resultados indican que la mayoría de las/los participantes no problematizó, de forma crítica, el papel de los medios de comunicación en la (re)construcción de la realidad social marcada por el género, apoyando la reificación de las tríades masculino-político-público y femenino-personal-privado. Más allá de eso, las/los participantes raramente cuestionaron los factores económicos, socioculturales y políticos que influyen en la producción informativa.

Conformado por una perspectiva feminista, este estudio destaca la necesidad de que se potencie la capacidad de agencia de los públicos de cara a las asunciones de género diseminadas por los medios informativos y se reflexione sobre los eventuales impactos de las representaciones mediáticas a nivel de la (re)configuración del espacio público.

\section{Palabras clave}

estudios de recepción, mujeres y política, representaciones sociales, estudios feministas de los medios.

\begin{abstract}
This paper examines reception practices of contents spread through the Portuguese newsmagazines "Sábado" and "Visão", exploring, in particular, the way young audiences understand, interpret and position themselves in relation to representations of women who hold political posts.

The thematic analysis conducted on the data resulting from a sequence of focus group discussions shows that participants tended to prefer readings that were aligned with traditional gender ideology. Despite occasional moments of negotiation, contestation and resistance, the results indicate that most participants did not critically problematize the role of the media in the gendered (re)construction of social reality, supporting instead the reification of the male-political-public and female-personal-private triads. Moreover, participants rarely questioned the economic, socio-cultural and political factors that exert influence on the news production.

Informed by a feminist perspective, this study therefore highlights the need to empower the audiences in relation to the gender assumptions that the news media have spread and to reflect about the impact of the media representations on the (re)configuration of the public sphere.
\end{abstract}

\section{Keywords}

reception studies, women and politics, social representations, feminist media studies. 


\section{Introducción}

Diversos estudios han demostrado la persistencia de representaciones estereotipadas a nivel de la producción de contenidos mediáticos (ej. Tuchman, 1979; Gallagher, 1981, 2006; van Zoonen, 1994; Álvares, 2006; Silveirinha, 2006; Cerqueira, 2012), que tienden a ser apropiadas de forma selectiva, (re)produciendo una visión androcéntrica (ej. Mendes \& Carter, 2008; Lobo \& Cabecinhas, 2010). Efectivamente, estos estudios permiten relativizar la tan propagada conquista de la igualdad de las mujeres en las esferas pública y privada, evidenciando que los medios de comunicación, más que entidades privilegiadas en la conducción del cambio social (concretamente por su papel de mediadores entre la realidad y las/los ciudadanas/os), se configuran frecuentemente como perpetuadores de asimetrías simbólicas (Amâncio \& Oliveira, 2006).

En el ámbito de los Estudios Feministas de los Medios, las prácticas de recepción han constituido un importante objeto de reflexión crítica (Watkins \& Emerson, 2000; Byerly \& Ross, 2006), a la par del análisis de las representaciones de género (Ross, 2010; Silveirinha, 2004) y del papel de las/los profesionales de los medios en la pervivencia de asimetrías sociales (Mendes \& Carter, 2008).

Desde la década de 1980, la investigación feminista ha intentado estudiar la recepción de contenidos difundidos por diferentes medios, tales como la televisión (Ross, 1995; Kim, 2006; Ferin-Cunha, 2007; Lobo \& Cabecinhas, 2010), las revistas femeninas (Winship, 1987; Mota-Ribeiro, 2010), los videojuegos (Walkerdine, 2006), las nuevas tecnologías móviles e Internet (Kim, 2007; Cerqueira, Ribeiro \& Cabecinhas, 2009).

Aunque se encuentren en creciente afirmación en las academias occidentales (Watkins \& Emerson, 2000), los estudios de recepción han dedicado una atención reducida al modo en el que los públicos negocian y se apropian de los contenidos informativos, descuidando el importante papel que los medios informativos (impresos) adquieren en la (re)construcción de significados y en la manutención de la ideología (tradicional) de género (van Zoonen, 1994).
Reconociendo el margen de autonomía e independencia de los individuos en relación a los mensajes mediáticos (Hall, 1980; Van Dijk, 2005), se propone en este estudio analizar las prácticas de recepción de contenidos difundidos por revistas portuguesas de información. En particular, se pretende estudiar el modo en el que los públicos jóvenes comprenden, interpretan y se sitúan en relación a las representaciones de mujeres que ejercen cargos políticos, observando la influencia de sus conceptualizaciones de género en la producción discursiva. En este sentido, se realizó una secuencia de once grupos focales con 101 estudiantes universitarias/os, cuyos datos textuales y respectivas inscripciones ideológicas fueron problematizados en el marco del Análisis Temático (Braun \& Clarke, 2006) partiendo de una perspectiva feminista.

A lo largo de las últimas décadas, los medios informativos han constituido un escenario privilegiado para la (re)configuración del espacio político y la mediación de la relación de la clase política con el electorado (Croteau, Hoynes \& Milan, 2012). Aunque diferentes tipos de medios produzcan efectos distintos, innumerables estudios muestran que la prensa está más fuertemente relacionada con la adquisición y la retención de la información política (Ross, 2003). En particular, abordando preferencialmente los llamados hard issues (Cardoso, 2009), las revistas de información tienen un papel crucial en la edificación de los modelos cognitivos y en la formación de opinión pública sobre asuntos políticos (Neuman, Just \& Crigler, 1992). Además, mediando quién adquiere importancia, cómo se expresa y representa públicamente, y qué es aceptado, naturalizado o polemizado, este tipo de medium colabora en la (re)construcción de los lugares de expresión pública e intercambio inmaterial, pudiendo influir en el ejercicio de la ciudadanía y en la configuración de las relaciones de género en el campo político.

En Portugal, la prohibición de la discriminación con base en el sexo quedó consagrada, en 1976, en el artículo $13^{\circ}$ de la Constitución de la República Portuguesa. Más tarde, impulsado por la ratificación 
de la Convención sobre la Eliminación de Todas las Formas de Discriminación contra las Mujeres $(\mathrm{CEDAW})^{1}$, en 1980, el Estado Portugués reconoció en la IV revisión constitucional de 1997 la importancia de las medidas de acción positiva como una respuesta sociopolítica a la prevalencia de desigualdades de género en diferentes áreas de actividad humana. La introducción de este tipo de medidas en el campo político tuvo lugar con la adopción de la Ley de la Paridad (Ley Orgánica $\mathrm{n}^{\circ}$. $3 / 2006)^{2}$, en 2006 , la cual se reflejó en una mayor visibilidad de las mujeres que ya integraban los partidos políticos (Santos \& Amâncio, 2012).

A pesar del aumento de la participación política de las mujeres en la última década ${ }^{3}$, la investigación científica ha demostrado que los medios (informativos) presentan una mediación de género en la cobertura de asuntos políticos, que puede afectar a las percepciones de los públicos/electorado en relación a la actuación de las mujeres, contribuyendo a la visión de la política como un espacio masculino (Ross, Evans, Harrison, Shears \& Wadia, 2013); la amenaza al mantenimiento eficiente del sistema democrático (Adcock, 2010); y la perpetuación de asimetrías sociales (Gallego, 2009).

De este modo, conviene analizar la capacidad de agencia de los públicos de cara a las asunciones de género difundidas por los medios informativos, pensar los eventuales impactos de las representaciones mediáticas a nivel de la (re)configuración del espacio público, así como anclar

\footnotetext{
${ }^{1}$ Adoptada por la Asamblea General de las Naciones Unidas el día 18 de diciembre de 1979, esta convención obliga en el artículo $4^{\circ}$ a los Estados miembros a implementar medidas temporales (especiales) dirigidas a la promoción de la igualdad de género en diferentes esferas de intervención.

${ }^{2}$ La Ley de la Paridad (Ley Orgánica n. 3/2006) estipula una representación mínima del 33\% de cada uno de los sexos en la composición de las listas para la Asamblea de la República, el Parlamento Europeo y las autarquías locales, así como establece que las listas no pueden contener más de dos candidatas/os del mismo sexo consecutivamente.

${ }^{3}$ De acuerdo con los datos del Inter-Parliamentary Union, la representación mundial de las mujeres en los parlamentos nacionales aumentó en la última década, pasando del 15,3\% en 2004 al 21,7\% en 2014. En Portugal, la representación parlamentaria de las mujeres es del $31,3 \%$, ocupando la $33^{\mathrm{a}}$ posición en el ranking mundial.
}

un conjunto de medidas necesarias para potenciar la cultura mediática y el ejercicio pleno de la ciudadanía. El presente estudio surge, así, de la necesidad de establecer en el ámbito de la crítica feminista una articulación entre los públicos, las mujeres y la política, intentando hacer frente a la escasez de estudios de recepción - bien a nivel nacional, bien a nivel internacional - que trascienden los llamados women's media/genres e inciden sobre los medios informativos. 


\section{Revistas de información, género y prácticas de recepción}

A pesar de la mayor presencia de las mujeres en las empresas de comunicación - incluyendo en las redacciones de las revistas portuguesas de información -, varios estudios han identificado una mediación de género en los procesos de producción noticiosa (Sreberny-Mohammadi \& Ross, 1996; Gidengil \& Everitt, 2003a), que tiende a consagrar la política como el espacio natural de los hombres y el de excepción para las mujeres (Ross et al., 2013).

A semejanza de otros medios de comunicación social (van Zoonen, 1994; Gallego, 2009; Gill, 2007; Martins, 2013), las revistas de información sustentan el predominio de la narrativa de lo masculino, fomentando la masculinización de la actividad política a través del mantenimiento de una jerga específica, de la menor visibilidad de las mujeres (políticas) en los medios, de la transmisión de representaciones de género esencialistas, y de la genderización de los contextos temáticos.

La imbricación globalizada de las asunciones de género en los medios informativos (Ross, 2010) puede promover en torno a los públicos la apropiación de significados apologéticos de relaciones de poder desiguales y de asimetrías sociales. Interviniendo en consonancia con los intereses de los grupos sociales dominantes (Mendes \& Carter, 2008), las industrias mediáticas promueven representaciones que tienen mayores probabilidades de ser aceptadas y pensadas como espejos de la realidad (Carter \& Steiner, 2004). En efecto, los medios informativos poseen un inconmensurable poder simbólico y persuasivo, con capacidad de influenciar las cogniciones sociales de los públicos (Van Dijk, 2005) y determinar los límites del conocimiento de la realidad social (Ross \& SrebernyMohammadi, 1997). Sin ser neutrales - particularmente en lo que respecta a las cuestiones de género (Sreberny-Mohammadi \& Ross, 1996) -, los medios influyen en el modo en el que los públicos perciben el universo político, evalúan los acontecimientos y efectúan sus deliberaciones electorales (Kahn \& Goldenberg, 1991; Kahn, 1994). Ya que la mayoría de los individuos no tiene experiencia directa con la política, su opinión sobre este dominio se forma significativamente a partir de los productos mediáticos (Kahn,
1994; Ross, 2003). Los públicos pueden, por consiguiente, asimilar los contenidos difundidos por los medios sin darse cuenta de la influencia que estos pueden ejercer en el modelaje de los acontecimientos: "el texto y el habla persuasivos pasan a no ser vistos como ideológicos, sino como verdades autoevidentes" (Van Dijk, 2005:82).

Sin embargo, conviene subrayar que los individuos no son marionetas, ausentes y pasivas, sino agentes activos en la construcción de significados (Asch, 1946; Hall, 1980; Kimmel, 2000; Van Dijk, 2005). Corroborando las teorías desarrolladas sobre todo a partir de la década de los ochenta - las cuales privilegian el análisis del modo en el que los públicos usan los medios y estudian su capacidad de agencia -, se sustenta que las/los lectoras/es solo en cierta medida se dejan influenciar por los contenidos mediáticos (Croteau et al., 2012; Ross, 2003).

Aunque la construcción social de la realidad tienda a seguir las ideologías dominantes en un determinado contexto cultural (Hall, 1980; Moscovici, 1981; Van Zoonen, 1994; Mendes \& Carter, 2008), los públicos pueden involucrarse - de forma activa, autónoma e independiente - en la (re)construcción de los significado(s) de los productos mediáticos: negociando, refutando y/o resistiendo (Van Dijk, 2005). La recepción constituye, por lo demás, una práctica que puede ser "capacitadora en vez de restringente, potenciadora en vez de opresiva, y activa en vez de pasiva” (Watckins \& Emerson, 2000:158). Además, influyendo en la (re)configuración de las dinámicas políticas (Hall, 1980), los medios pueden desempeñar un papel muy positivo en el mantenimiento de la democracia en las sociedades contemporáneas, capacitando a los públicos para que estos asuman sus derechos de participación política (Ross, 2003).

La variabilidad en las prácticas de recepción de contenidos mediáticos - concretamente de aquellos de fondo político (Ross, 2003) - tiene relación directa con factores estructurales, socioculturales e individuales (Croteau et al., 2012; van Zoonen, 1994), tales como el contexto en el cual el/la receptor/a se encuentra, el sexo, la edad, el estatus social y sus expe- 
riencias anteriores (Gamson, Croteau, Hoynes \& Sasson, 1992; Ross, 2003). Por eso, la realidad construida por los medios puede ser aceptada o refutada, pudiendo o no influenciar, de forma determinante, la interpretación de los públicos acerca de un determinado acontecimiento (Hall, 1980; Gamson et al.,1992).

En resumen, de acuerdo con Silveirinha (1998:9), es necesario analizar el "papel de la comunicación en la vida política y del espacio en el que se intercambian los discursos discrepantes de los actores que, en democracia, tienen oportunidad de expresarse públicamente". Por otro lado, debemos investigar los mecanismos a través de los cuales los públicos comprenden las asunciones de género imbricadas y los contenidos acerca de intervinientes políticos. Las investigaciones en este ámbito permitirán mostrar el papel de los medios en la constitución de mujeres y hombres como sujetos políticos, interrogando eventuales diferencias, impactos, cambios y desafíos.

\section{Metodología}

El presente estudio cualitativo fue conducido durante el mes de octubre de 2012 con el objetivo de analizar las prácticas de recepción de contenidos difundidos por las revistas portuguesas de información generalistas con mayor tirada en el contexto nacional (Marktest, 2009): las revistas Visão y Sábado. En particular, se intentó estudiar las interpretaciones y los posicionamientos de los públicos (jóvenes) de cara a las representaciones mediáticas de mujeres que ejercen cargos políticos.

En términos metodológicos, se optó por la realización de grupos focales, ya que estos comportan innumerables ventajas para los estudios de recepción (Mendes \& Carter, 2008; Lobo \& Cabecinhas, 2010). En el conjunto de sus principales ventajas, destaca la mitigación de los problemas éticos relacionados con el poder ejercido por el/la investigador/a en el momento de la recogida de datos, la posibilidad de analizar las co-construcciones del significado en las prácticas de interacción social y el hecho de que muchas veces constituyan una experiencia que se vive como positiva por las/los participantes (Munday, 2006; Wilkinson, 1998). Como refiere Morgan (1998:9), "los grupos focales son fundamentalmente un modo de escuchar a las personas y de aprender con ellas. Los grupos focales crean líneas de comunicación”.

En los grupos focales realizados, que tuvieron lugar en las instalaciones de la Universidade do Minho, en Portugal, participaron 101 estudiantes universitarias/os, de las/los cuales 77 elementos eran del sexo femenino $(76,2 \%)$ y 24 del sexo masculino $(23,8 \%)$, con una media de edades de 20 años. Las/los participantes cursaban las licenciaturas de Ciencias de la
Comunicación $(55,4 \%$ ) y de Sociología (42,6\%), y fueron distribuidas/os en once grupos focales.

El guion fue desarrollado de acuerdo con cuestiones semiestructuradas, previamente elaboradas, que se centraron en dos temas principales: a) usos sociales de las revistas de información generalista; b) percepciones acerca de las representaciones de género en las revistas de información.

En una fase intermedia de la realización de los grupos focales, fueron distribuidos entre las/los participantes dos reportajes remisivos a cuestiones políticas, a saber: "Las rebeldías de la protegida de Portas", publicado en la revista Sábado (n ${ }^{\circ}$ 372, 16 de junio de 2011), y "Ahora sí que son ellas", publicado en la revista Visão (n. ${ }^{\circ}$ 957, 7 de julio de 2011). Este procedimiento tenía como objetivo servir de estímulo al intercambio de ideas y su profundización y, en algunos casos, romper eventuales bloqueos o impases en la discusión.

Posteriormente, fue solicitado a las/los participantes que analizasen ambos trabajos periodísticos, teniendo en cuenta las representaciones de mujeres con intervención en la esfera política/pública.

Finalmente, en el caso de haber sido reconocidas asimetrías de género en los medios durante el debate, las/los participantes fueron invitadas/os a debatir sobre las políticas públicas de promoción de la igualdad de género y sobre las áreas que consideraban prioritarias en la actualidad.

Terminado el debate, se agradecía a las/los participantes y se demostraba la disponibilidad del equipo para responder a eventuales cuestiones, en ese momento o posteriormente. De media, las sesiones de los diversos grupos focales tuvieron una duración aproximada de 90 minutos. 
El material obtenido a través de la realización de los grupos focales fue tratado recurriendo al software NVivo 8.0. Se optó por realizar un análisis temático (Braun \& Clarke, 2006) del material, ya que este tipo de procedimiento permite identificar y analizar patrones (temas) en los datos, potenciando la comprensión de los significados asociados a datos textuales (Guest, Macqueen \& Namey, 2012).

De este modo, en este artículo presentamos el análisis de los paradigmas representacionales identificados por las/los participantes durante los grupos de discusión a fin de observar sus interpretaciones y posicionamientos. Tal análisis requirió la constitución de narrativas temáticas que - agregando los temas (in)directamente relacionados y confiriendo una observación concertada de los significados semánticos a nivel micro y macro - permitieron interpelar la fluidez, las ambigüedades y las vacilaciones discursivas.

\section{Análisis y discusión de los paradigmas representacionales identificados}

En los grupos de discusión ${ }^{4}$, la mayoría de las/los participantes reconoció que las desigualdades de género continúan existiendo en Portugal, concretamente en la esfera política. Con todo, no se atribuyó una particular relevancia a su dimensión, manifestaciones y efectos políticos, económicos y socioculturales. Dando énfasis a las consecuciones verificadas en el escenario de los derechos de las mujeres en las últimas décadas, las/los participantes describieron la discriminación contra las mujeres a partir de un prisma optimista y no-problematizante, en un abordaje que tendió a validar los presupuestos de la ilusión de la igualdad (Banyard, 2010).

“... creo que ahora ya no hay aquella discriminación inicial en la que las mujeres no pueden hacer nada más”. (Rita Mendes, 18 años)

Cuando se les cuestionó sobre las causas del mantenimiento de las desigualdades de género en la esfera pública/política, las/los participantes presentaron principalmente aspectos de naturaleza histórica y sociocultural. Con todo, surgieron además discursos que destacaron la posibilidad de que las mujeres no posean las características necesarias para la intervención política.

“... los géneros tienen diferencias entre sí, entonces tal vez [la capacidad de liderar y la idoneidad para ejercer cargos políticos] sean características más encontradas en

\footnotetext{
${ }^{4}$ Para garantizar el anonimato y la confidencialidad de los datos, los nombres de las/los participantes mencionados en este artículo son ficticios.
}

hombres que en mujeres...”. (António Pereira, 25 años)

Algunas/os participantes atribuyeron la responsabilidad a las propias mujeres, habiéndoles imputado complicidad con prácticas de desigualdad ejercidas contra sí mismas, así como desinterés y miedo en relación a la intervención en áreas convencionalmente dominadas por hombres.

“... el problema también ya es de las mujeres que aceptan y que llevan toda la carga de los hombres...”. (Luciana Ventura, 35 años)

"Yo creo que, como antes eran privadas de eso, quizás ahora tienen un poco de miedo de arriesgar...”. (Leonor Pedrosa, 18 años)

Mientras que la escasez de mujeres en la esfera pública fue principalmente atribuida a actitudes y comportamientos de estas (sin tener en cuenta la influencia de factores estructurales), la infraparticipación de los hombres en la esfera privada (asumida como área convencionalmente asociada a las mujeres) fue justificada como consecuencia de la actuación de agentes ideológicos, como la familia y la educación. Además de eso, contrariamente a los de las mujeres, los esfuerzos de los hombres para igualar los papeles de género fueron significativamente valorados. Aunque la mayoría de las/los participantes haya señalado la persistencia de situaciones de desigualdad de género en la sociedad portuguesa, cuando el centro de la discusión se dirigió sobre los medios, estos posicionamientos tendieron a alterarse. En realidad, los discursos producidos manifestaron una cierta renuencia en reconocer el mante- 
nimiento de asimetrías de género en las industrias mediáticas y a través de los medios, habiendo favorecido la idea de que la producción de contenidos se pauta exclusivamente por principios de imparcialidad y objetividad (Byerly \& Ross, 2006).

"Yo creo que a pesar de que haya alguna discriminación contra las mujeres en Portugal, eso no se debe al papel de los medios de comunicación. Creo que estos intentan mantener la imparcialidad que les es exigida...”. (Patrícia Castro, 18 años)

Algunas/os participantes consideraron la discriminación con base en el sexo menos grave que otras formas de discriminación, concretamente la discriminación racial o étnica. Esta jerarquización de diferentes formas de discriminación revela la escasez de conocimiento y la ausencia de conciencia crítica sobre la amplitud de las asimetrías simbólicas en el contexto mediático portugués. Además, contradice la evidencia empírica en el campo de los estudios de la percepción humana, que señala un mayor impacto de la discriminación con base en el sexo comparada con la racial (ej. Cabecinhas \& Amâncio, 1999).

“... creo que se nota más [discriminación en los medios] en términos de etnia y de raza que propiamente en términos de sexo". (César Pinto, 19 años)

En términos generales, las asimetrías de género en los medios fueron eminentemente asumidas como una no-cuestión, no habiendo sido consideradas como un posible criterio de evaluación de la calidad periodística (Lobo \& Cabecinhas, 2010). Exceptuando algunas observaciones acerca de las diferencias entre mujeres y hombres a nivel de la representación cualitativa - las cuales, por lo demás, pueden haber sido influenciadas por el material-estímulo usado durante los grupos de discusión -, las desigualdades de género no constituyeron objeto de especial interés, preocupación y/o reflexión crítica.

En una dinámica transversal a los grupos de discusión, las/los participantes identificaron y exploraron los paradigmas en los cuales las mujeres con incumbencias políticas son representadas en los medios informativos, a saber: falta de preparación y necesidad de un mentor $(35,6 \%)$, vida privada y ámbito doméstico $(22,9 \%)$, mujeres como instrumentos políticos
$(13,4 \%)$, participación creciente en la esfera política $(10,2 \%)$, conciliación familia-trabajo $(8,8 \%)$, excepcionalidad $(7,0 \%)$ y apariencia física y cuerpo $(2,1 \%)$. A continuación, se analizan los modos de representación identificados por las/los participantes a partir de su inclusión en tres narrativas temáticas: participación de las mujeres en la política; vida privada y ámbito doméstico; y apariencia física y cuerpo.

\subsection{Participación de las mujeres en la política}

Innumerables participantes consideraron que las mujeres que intervienen en la esfera política - en calidad de jefes de Estado, miembros del Gobierno, diputadas de la Asamblea de la República, etc. - tienden a ser asociadas con características como la incompetencia profesional, la indefinición de intereses y la ausencia de convicciones (políticas) independientes. Son representadas como individuos cuya actuación en el espacio público/político se debe y depende de sus mentores, de sus congéneres hombres.

Fue justamente esta la idea que las/los participantes tuvieron durante una de las piezas presentadas para discusión en grupo, en la cual la actual ministra de la Agricultura y del Mar, Assunção Cristas, y el presidente del partido CDS-PP, Paulo Portas, figuraban como protagonistas (Visão, n. ${ }^{\circ}$ 957). Mientras que la primera surgió como desprovista de la preparación necesaria para ejercer el cargo político que le había sido atribuido, el segundo emergió como la figura que define el recorrido de la ministra, asegura protección y hace el papel de mentor político.

\footnotetext{
“(...) más que una figura en la cual ella [Assunção Cristas] puede verse, [Paulo Portas] es alguien que está por detrás manejando las marionetas". (Joana Silva, 21 años)

“(...) la idea de que ella [Assunção Cristas] es una niña y que él [Paulo Portas] solo está ahí para protegerla porque pobrecita... está ahí un poco perdida”. (Luís Fonseca, 19 años)
}

Además del texto, también los elementos visuales fueron objeto de análisis por parte de las/los participantes. La imagen de la pieza - en la cual Assunção Cristas aparece con la cabeza en el hombro de Paulo 
Portas, que la acaricia - fue tendencialmente interpretada como una alusión a una relación de padre e hija.

“(...) ella [Assunção Cristas] parece la hija querida de papá”. (Miguel Ribeiro, 20 años)

Aunque no se base en una crítica fundamentada, la posición de las/los participantes señala la imbricación en los medios informativos de abordajes esencialistas (Gidengil \& Everitt, 2003a; Ross, 2004; Gill, 2007) y del paternalismo protector/sexismo benevolente (Glick \& Fiske, 1996).

No pocas veces, la autonomía y la idoneidad (política) de las mujeres son puestas en duda, legitimando las relaciones jerárquicas tradicionales: los hombres como mentores, las mujeres como figuras que necesitan un mentor. Mientras que los primeros son a priori considerados idóneos para el ejercicio de funciones políticas, las mujeres son puestas bajo investigación periodística, teniendo que emprender esfuerzos redoblados para legitimar su estatuto.

Históricamente, las mujeres han permanecido como ciudadanas de segunda clase: la (progresiva) conquista de sus derechos de ciudadanía ha ido acompañada por procesos rígidos de binarización porque son consideradas individuos con características divergentes de las del modelo universal de persona adulta: autónoma, racional e independiente (Amâncio, 1998). La reducción de la diversidad de (inter)subjetividades, identidades y experiencias a un único modelo de ciudadanía tiene, para Lister (1997), un propósito principal: la reificación de valores androcéntricos en las estructuras sociopolíticas, o sea, la institución como principal característica del individuo-ciudadano de normas que son convencionalmente definidas como masculinas (p.ej. imparcialidad, independencia, agencia política, etc.). Estas configuraciones ideológicas expresan, por lo demás, el mantenimiento de la lógica de la identidad que - radicada en el núcleo de la retórica universalista - reprime y anula la(s) diferencia(s) (Young, 1990), favoreciendo la exclusión de las mujeres de la esfera política.

Coexistiendo con la representación de las mujeres como no preparadas y con necesidad de mentor (político), varias/os participantes indicaron la existencia de otro paradigma representacional: el de las mujeres como instrumentos políticos. De forma destacable, las/los participantes señalaron el hecho de que las revistas portuguesas de información representasen la entrada y la participación de las mujeres en la política como una estrategia dirigida al fortalecimiento de la imagen del partido junto al electorado, sobre todo en periodos electorales.

“(... él [Paulo Portas] apuesta por las mujeres y las usa tal vez como una estrategia electoral ...”. (João Almeida, 21 años)

Para algunas/os participantes, las mujeres emergieron especialmente como maniobras políticas que los partidos utilizan para atraer el apoyo de las mujeres electoras.

"Ellos hacen de ella [Assunção Cristas] como si fuese un esquema para conseguir más votos, sobre todo por parte de las mujeres...”. (Margarida Oliveira, 20 años)

Esta observación es, por lo demás, congruente con la investigación feminista desarrollada sobre las dinámicas, las relaciones y las (inter)dependencias que caracterizan el campo político. Véase, por ejemplo, el estudio de Heldman, Oliver y Conroy (2009): analizando la cobertura mediática de Geraldine Ferraro y Sarah Palin, las autoras concluyeron que las mujeres candidatas a posiciones de relevancia política - como la vice-presidencia - son escogidas con el objetivo primero de conquistar el apoyo de las mujeres (votantes) y, por consiguiente, aumentar las opciones electorales de los respetivos partidos.

En una exposición de tendencia corroborante, un número considerable de participantes destacó que en este paradigma representacional subyacen dos postulados esenciales: el de que existe una identificación de género, según la cual las mujeres tenderán a votar a candidatas; y el de que las mujeres - como grupo social - poseen un menor interés en relación a la política y, en esa medida, necesitan ser conquistadas.

“(...) al llamar a tantas mujeres para colaborar con él, [Paulo Portas] está dirigiéndose a la población femenina que, por lo general, está más desvinculada del mundo de la política”. (Rute Santos, 19 años)

Sin embargo, hay estudios que han demostrado que la identificación de género no constituye per se un factor 
explicativo de las preferencias partidistas del electorado, concretamente de las mujeres (ej. Dolan, 2004). Aunque estas manifiesten una mayor propensión para votar a candidatas, esta correlación no es lineal ni aplicable a todas las circunstancias electorales: intervienen, igualmente, variables como la identificación con el partido político (Dolan, 2004). Además, las intenciones de voto pueden también estar influenciadas por estereotipos de género. Por lo general, las electoras no reconocen en las candidatas la capacidad de liderazgo político, pues no asocian este trazo de personalidad a las dimensiones del estereotipo femenino (Amâncio, 1998).

Además, las mujeres figuran como individuos debutantes en la esfera política: su intervención pertenece al tiempo reciente - al de ahora. Es, justamente, este paradigma el que varias/os participantes señalaron en las revistas portuguesas de información e - inclusive - corroboraron, reforzando la invisibilidad histórica y política de las mujeres.

“(...) lo que más podemos observar es el aumento de las mujeres a la política...”. (Pedro Rodrigues, 20 años)

“(...) habla del hecho de que, ahora, vengan muchas mujeres a la política...”. (Rita Mendes, 18 años)

Diversas investigadoras sostienen que la representación de las mujeres como novedades sitúa sus discursos y acciones bajo especial escrutinio (ej. Ross, 2004; Lobo \& Cabecinhas, 2010). De forma recurrente, la atribución de un carácter debutante a la participación de las mujeres en la política surge del mantenimiento del paradigma de la primera mujer (ej. Heldman, Oliver \& Conroy, 2009). Este tipo de abordajes podrá limitar las consecuciones (electorales) de las mujeres en el escenario político y reforzar imaginarios androcéntricos (Gigengil \& Everitt, 2003b). Figurando eminentemente como personas que se estrenan en el campo político, las mujeres tenderán a ser vistas como intrusas en un territorio de natural pertenencia a los hombres (Braden, 1996; Cabrera, Flores \& Mata, 2012).

En una aparente corroboración de la ideología meritocrática, las/los participantes mencionaron además el hecho de que las mujeres figuren en las revistas portuguesas de información como individuos excepcionales que, a través de esfuerzos personales, consi- guieron navegar en un campo dominado tradicionalmente por hombres - la política.

"Yo creo que ellos [periodistas] quieren dar la imagen de supermujer...”. (Eduarda Martins, 20 años)

"Creo que es para mostrar que ella [Assunção Cristas] tuvo coraje y firmeza. Y que asumió un cargo que, quizás, no era exactamente aquello que ella quería y aquello que ella dominaba, pero que lo asumió con coraje...”. (César Pinto, 19 años)

Efectivamente, los medios informativos favorecen, en numerosas ocasiones, el modelo de mujeresexcepción (Cerqueira, 2012), que tiende a estructurarse en disonancia con las características atribuidas a la feminidad normativa. En los grupos de discusión aunque algunos discursos hayan señalado, de forma esporádica, la fusión entre los trazos de feminidad y de masculinidad (hegemónicas) -, varias/os participantes destacaron, justamente, el hecho de que las mujeres se hubiesen caracterizado en base a atributos asociados al estereotipo masculino, tales como la capacidad de liderazgo, el control y la supervisión (Amâncio, 1998).

“... habla de ella [Assunção Cristas] como un ejemplo: "es este el ejemplo de las personas que van a dirigir"”. (Catarina Lemos, 20 años)

"La idea que se tiene de una mujer cuando tiene un problema es la de que se queda por casa... quien va para el campo de batalla incluso así son, supuestamente, los hombres... la bicicleta [con la cual Assunção Cristas aparece en una fotografía] presupone un espíritu aventurero mientras que la idea de lo femenino es lo contrario...”. (Jorge Peixoto, 22 años)

Ya que las dimensiones del estereotipo masculino coinciden, por convención histórica, con aquellas asociadas al paradigma universalista de la ciudadanía (política), la atribución a las mujeres de trazos da masculinidad hegemónica puede ser indicio de que estas tan solo adquieren relevancia (mediática) y excepcionalidad cuando corresponden al modelo masculino/normativo. Tales aproximaciones confirman el posicionamiento de Young (1990): los grupos sociales cuyos atributos se consideran a priori como 
divergentes de aquellos que fueron instituidos como norma son comúnmente objeto de estrategias de asimilación. Esta coacción asimilacionista tenderá a anular la diversidad de identidades, favoreciendo la universalización y la neutralización de los intereses, necesidades y experiencias de los grupos dominantes y su consagración como el referente simbólico. En otras palabras, redundará en la legitimación de relaciones jerárquicas, en el ejercicio desigual del poder $\mathrm{y}$, sobre todo, en la perpetuación de la opresión social (de los miembros) de determinados grupos sociales.

Además, descentrando la atención de las asimetrías que atraviesan el campo político para enfatizar las conquistas individuales de las mujeres, los medios (informativos) tienden a perpetuar el discurso esencialista-individualista (Nogueira, 2006). Como fue referido puntualmente por las/los participantes, las mujeres surgen como detentoras de un estatus simbólico (token status) (Kanter, 1977) que - a pesar de los obstáculos de naturaleza diversa y a través de su esfuerzo individual - obtuvieron éxito en sus proyectos profesionales/políticos.

“... vemos a Assunção Cristas como una especie de supermujer, que consiguió llegar al Gobierno, que hizo un montón de cosas importantes y tuvo éxito...”. (Patrícia Castro, 18 años)

Este tipo de paradigma representacional no solo contribuye al mantenimiento de las categorías dicotómicas femenino y masculino, reforzando relaciones de poder desiguales, como también apoya una retórica individualista (Nogueira, 2006) que esconde los obstáculos socioculturales e institucionales que limitan la participación política de las mujeres y fomentan contextos de opresión y de injusticia social (Young, 1990).

\subsection{Vida privada y ámbito doméstico}

Además de la participación de las mujeres en la política, la vida privada y el ámbito doméstico constituyeron igualmente una importante narrativa temática, compilando asunciones semánticas muy pertinentes desde el punto de vista feminista.

A semejanza de estudios anteriores (ej. Kahn \& Goldenberg, 1991; Kahn, 1994; Norris, 1997; Ross,
2010), innumerables participantes afirmaron que las revistas portuguesas de información generalista tienden a explorar aspectos relacionados con la vida privada de las mujeres políticas - como sus relaciones familiares y estado civil - y a remitirlas al espacio doméstico, esbozando frecuentes menciones al ejercicio de la maternidad y a la prestación de cuidados en la familia.

“... aquí muestra una mujer más humanística, una mujer que va a la iglesia, una mujer que quiere pasar tiempo con la familia, una mujer que tiene el horario de trabajo de $x$ a $x$ horas $y$, a partir de ahí, está con su familia. El trabajo no es prioritario. Creo que da la idea de la mujer como ama de casa". (Carlos Neves, 18 años)

Por otro lado, como fue mencionado durante los grupos de discusión, los hombres políticos no son, por lo general, observados en lo que respecta a su actuación en la vida privada/doméstica - particularmente sobre el ejercicio de la paternidad y de sus relaciones de parentesco (Braden, 1996) -, así como sobre el modo de conciliar la vida familiar con las responsabilidades profesionales.

“(...) si fuese un hombre, no estarían aquí hablando de los hijos...”. (Filipa Cunha, 20 años)

“(...) nunca se vio que se hablase de la vida privada de un hombre político, decir que él pidió que adelantasen las reuniones a la comida porque tenía que cenar con la familia...”. (Catarina Lemos, 20 años)

La desacreditación de las mujeres como sujetos de acción/intervención política - que se potencia por la indagación en su vida privada - se refleja especialmente en la genderización de los contextos temáticos en los cuales son representadas por los medios (Kahn \& Goldenberg, 1991; Kahn, 1994).

Independientemente de la posición que ocupen en el ámbito político (ej. jefes de Estado, miembros del Gobierno, diputadas de la Asamblea de la República, etc.), las mujeres tienden a surgir como fuentes de información en temáticas que están relacionadas o constituyen una extensión de las actividades vinculadas convencionalmente a la esfera doméstica/privada. Por lo general, aparecen en los medios informativos para opinar sobre los conocidos como soft issues, tales como 
la educación y el medio ambiente (Kahn \& Goldenberg, 1991; Kahn, 1994), la salud (Gill, 2007) y las cuestiones de género (Ross et al., 2013). Por otro lado, los hombres surgen representando predominantemente áreas como la economía y finanzas, la defensa nacional o la diplomacia, entre otros (Kahn, 1994). Y, emergiendo como actores naturales en estas esferas, se les atribuyen trazos de personalidad relevantes en el contexto profesional, incluyendo la exigencia, la seriedad, el espíritu crítico y el dinamismo (Ross \& Sreberny-Mohammadi, 1997; Gidengil \& Everitt, 2003a).

Resultando de la actuación de la ideología de género y del mantenimiento de la asimetría simbólica (Amâncio \& Oliveira, 2006) - que radican en la cultura periodística y en las industrias mediáticas -, el énfasis en la vida privada/doméstica de las mujeres ejerciendo cargos políticos refuerza la no-pertenencia de estas al espacio público y su inhabilidad para el ejercicio serio de esas funciones (Kahn \& Goldenberg, 1991; Ross, 2010), así como garantiza y valida los papeles tradicionales de género (Braden, 1996), pudiendo afectar negativamente a su repercusión política de cara al electorado.

\subsection{Apariencia física y cuerpo}

En una convergencia con la investigación feminista (ej. Gallagher, 2001; Gidengil \& Everitt, 2003a; Ross, 2010), algunas/os participantes mantuvieron que las mujeres políticas fueron, de forma significativa, representadas en las revistas portuguesas de información generalista partiendo de su apariencia física y cuerpo.

“(...) en la cuestión de la imagen, la mujer tiene que esforzarse más, a ella se le exige mucho más que a los hombres...”. (António Pereira, 25 años)

“(...) yo me acuerdo de cuando la ... Ministra de Agricultura fue a dar una conferencia con un vestido corto $y$ hubo enseguida muchas discusiones". (Carolina Torres, 18 años)

Los medios informativos tienden, de hecho, a dejar en segundo plano lo que las mujeres piensan y dicen, dando prioridad, al contrario, a aspectos relacionados con su indumentaria, sentido de la moda, etc. Esta tónica en la apariencia física (que, no pocas veces, tangencia la sexualización) se aplica a las mujeres como grupo social, independientemente de su background, profesión y ámbitos de actuación (Ross, 2004).

Aunque ejerzan cargos de (asumida) relevancia pública, las mujeres políticas están sujetas, en igual grado y frecuencia que las demás, a procesos de sexualización/objetificación (Ross \& Sreberny-Mohammadi, 1997). Desde el uso del lenguaje a los contenidos fotográficos, los medios recurren a diferentes estrategias para restaurar la feminidad normativa y constituir a las mujeres como heraldos de la belleza occidentalmente establecida. Como refiere Gill (2007:117), los abordajes sexualizadores de las mujeres tienen objetivos específicos: "son parte de la operación de poder que trivializa las perspectivas de las mujeres y las mantiene 'en su lugar'”.

Por otro lado, a diferencia de sus congéneres, los hombres políticos raramente aparecen referidos partiendo de sus atributos físicos. Para las/los participantes, los hombres fueron especialmente relacionados con sus opiniones, acciones y comportamientos, aunque estos puedan ser socialmente cuestionables y disparar polémicas.

“(...) la mujer tiene mucha más obligación de ser guapa que el hombre... Retrataron a Lula [da Silva] porque a él le gustaba beber cachaza. Ahora, en el caso de Dilma [Rousseff], es porque ella es fea... esa cosa de ser fea creo que recae mucho más en la mujer”. (Paula Barbosa, 21 años)

Secundando a Baudrillard (1970/2010) y Wolf (1992), la asociación con los patrones de belleza constituye un imperativo solo para las mujeres, sin imponerse con igual urgencia a los hombres. Convergiendo con las dimensiones del estereotipo femenino (Amâncio, 1998), este tipo de representación confirma, por lo demás, las afirmaciones de Gallego (2009:45): "Ellas [las mujeres] son el objeto observado, que no forman parte del centro a partir del cual se observa y se narra. De ahí que las mujeres sean presentadas por lo que son, no por lo que hacen”. Por el contrario, los "hombres son el verbo, y el verbo es acción": son los sujetos que actúan y protagonizan los acontecimientos, a partir de los cuales se narra todo.

La importancia atribuida a la apariencia física de las mujeres tiene subyacentes asunciones ideológicas (de 
género) más ampliamente instituidas, es decir, la asociación de patrones específicos de belleza a la feminidad normativa. Teniendo la función de coerción social, el mito de la belleza constituye per se la expresión última de la dominación masculina: colabora en el mantenimiento de relaciones de poder desiguales, movido por el propósito de "destruir psicológicamente y con subterfugios todo lo positivo que el feminismo proporcionó a las mujeres material y públicamente" (Wolf, 1992:13-15).
Apoyada en una connotación valorativa diferenciada, donde el sexo masculino ocupa una posición dominante y el femenino surge en una posición subordinada (Amâncio, 1998), este tipo de paradigma representacional contribuye al descrédito de las opiniones, experiencias y actuaciones profesionales de las mujeres, limitando sus potencialidades y consecuciones en la esfera política (Ross, 2010).

\section{Consideraciones finales}

En línea con el compromiso con la investigación del cambio sociopolítico y la promoción de una sociedad más inclusiva, la observación de las prácticas de recepción ha adquirido una creciente importancia en el ámbito de los Estudios Feministas de los Medios (Mendes \& Carter, 2008). Además de comprobar la variabilidad interpretativa de los públicos en relación a los productos mediáticos, la crítica feminista de la recepción ha contribuido a la desnaturalización de las diferencias de género, mostrando su carácter variable y dinámico (Carter \& Steiner, 2004).

En el presente estudio, el análisis temático del material obtenido tras la realización de once grupos focales - en el que participaron 101 estudiantes portuguesas/es de Enseñanza Superior - permitió investigar las prácticas de recepción de contenidos noticiosos. En particular, posibilitó la observación del modo en el que los públicos (jóvenes) interpretan y se sitúan de cara a los paradigmas representacionales en los cuales las mujeres que ejercen cargos políticos surgen en los medios, en general, y en las revistas de información generalista, en particular.

Los paradigmas representacionales identificados por las/los participantes fueron encuadrados en tres narrativas temáticas: participación de las mujeres en la política; vida privada y ámbito doméstico; y apariencia física y cuerpo.

Aunque hayan surgido puntualmente posiciones críticas, la mayoría de las/los participantes no cuestionó el carácter ideológico de las representaciones mediáticas de las mujeres políticas, concretamente de aquellas que las conforman como no preparadas y con necesidad de mentores. Además, a pesar de haber señalado el paradigma que atribuye excepcionalidad a las mujeres, las/los participantes tendieron a secun- dar la retórica individualista que - sustentada por la ideología meritocrática - esconde los obstáculos institucionales y socioculturales a la participación política de las mujeres. Una parte significativa de las/los participantes no observó, de forma profunda, las asunciones que están implícitas a los paradigmas representacionales identificados, es decir, el descrédito de la idoneidad política y de la autonomía profesional de las mujeres, el paternalismo protector/sexismo benevolente, así como la legitimación social de relaciones jerárquicas en la esfera política.

Destacamos, con todo, la existencia de discursos críticos de las referencias que las revistas portuguesas de información generalista dirigieron a la vida privada y a aspectos de la esfera doméstica de las mujeres políticas, así como a su apariencia física y cuerpo. Innumerables participantes destacaron, a ese respecto, la prevalencia de diferencias entre mujeres y hombres en lo que concierne a las representaciones mediáticas, destacando que - contrariamente a sus colegas -, las mujeres políticas son observadas al nivel de la prestación de cuidados a terceros, ejercicio de la maternidad, conjugación de la familia con las responsabilidades profesionales y forma de vestir, entre otros.

Los resultados generales muestran que el mantenimiento de asimetrías de género en los medios informativos, al nivel de la representación cualitativa, no se considera por los públicos (jóvenes) como una cuestión de relevancia significativa. A pesar de los momentos puntuales de negociación, controversia y resistencia en relación a los significados difundidos por los medios, la mayoría de las/los participantes adoptó posiciones tranquilizadoras que inhibieron una concienciación individual/social acerca de las conse- 
cuencias del mantenimiento de relaciones de poder desiguales en los medios, en la esfera política y en la sociedad. Este estudio demuestra, así, que los públicos producen sobre todo lecturas congruentes con ideologías y discursos hegemónicos, como los de género, los cuales, por lo demás, tienden a ser los puntos de vista que prefieren los medios informativos (ej. Sreberny-Mohammadi \& Ross, 1996; Gidengil \& Everitt, 2003; Ross, 2004; Lobo \& Cabecinhas, 2010).

En una apreciación global, puede afirmarse que la principal contribución de este estudio consiste en analizar las (inter)relaciones entre los públicos, medios informativos, género y representación cualitativa, destacando la importancia de promover la reflexión sobre los posibles impactos que las representaciones mediáticas tienen a nivel de la (re)configuración del espacio político/público y en el ejercicio de la ciudadanía de forma plena, diversa e inclusiva.

Independientemente de las contribuciones emprendidas en este estudio, hay que resaltar que los resultados aquí presentados no son extrapolables. Proporcionan tan solo indicaciones preliminares acerca del modo en el que los públicos interpretan y se sitúan en relación a las representaciones mediáticas y, en particular, a las asunciones de género difundidas por los medios informativos. Una investigación más profunda exigirá la participación de un mayor número de intervinientes y mayor diversidad, concretamente a nivel de la edad, identidad de género, nivel socioeconómico, nivel de estudios, áreas de formación profesional, etc. Además de eso, es necesario garantizar una ma- yor variedad en términos de material-estímulo - ya que este podrá influenciar los discursos producidos y la fundamentación de las posiciones ideológicas -, así como potenciar el cruce de diferentes aproximaciones teórico-metodológicas.

En resumen, se impone la necesidad de profundizar la investigación científica sobre prácticas de recepción con vistas al empoderamiento semiótico de los públicos (jóvenes) en relación a los productos mediáticos y a los contextos socioculturales, económicos y políticos en los cuales estos son creados (Hobbs, 2005), así como al impacto de estos en la (re)construcción de la realidad social y a los mecanismos por los cuales los contenidos pueden ser refutados y/o modificados (Gallagher, 2001; Silverblatt, 2001). Partiendo de una intervención concertada en la producción y en la recepción (Kellner, 1995), la educación feminista para los medios tiene particular importancia para la deconstrucción de las asunciones de género (Gallagher, 2001; Teurlings, 2010). A través de iniciativas como la formación de profesionales de los medios para las cuestiones de la diversidad y la inclusión de perspectivas feministas en los currículos universitarios -, la promoción de la alfabetización (trans)mediática tiene la potencialidad de conferir a todos los agentes involucrados (tanto en la producción como en la recepción) una comprensión más amplia de los significados imbricados en la compleja tríade políticamedios-públicos y una mayor concienciación de lo mucho que hay que hacer aún para eliminar las desigualdades de género en las sociedades contemporáneas.

\section{Referencias}

- Adcock, C. (2010). The Politician, the Wife, the Citizen, and Her Newspaper. Feminist Media Studies, 10(2), 135-159.

- Álvares, C. (2006). Feminismo e Representação Discursiva do Feminino: a Presença do Outro na Teoria e na Prática. Ex aequo, 14, $35-43$.

- Amâncio, L. (1998). Masculino e Feminino: a Construção Social da Diferença. Lisboa: Edições Afrontamento.
- Amâncio, L. \& Oliveira, J. M. (2006). Men as Individuals, Women as a Sexed Category: Implications of Symbolic Asymmetry for Feminist Practice and Feminist Psychology. Feminism \& Psychology, 16(1), 35-43.

- Asch, S. E. (1946). Forming Impressions of Personality. The Journal of Abnormal and Social Psychology, 41(3), 258-290.

- Banyard, K. (2010). The Equality Illusion, The Truth about Women and Men Today. Londres: Faber \& Faber. 
- Baudrillard, J. (1970/2010). A Sociedade de Consumo. Lisboa: Edições 70.

- Braun, V. \& Clarke, V. (2006). Using Thematic Analysis in Psychology. Qualitative Research in Psychology, 3(2), 77-101.

- Braden, M. (1996). Women, Politics and the Media. Lexington: University Press of Kentucky.

- Byerly, C. \& Ross, K. (2006). Women and Media: A Critical Introduction. Malden, MA: Blackwell Publications.

- Cabecinhas, R. \& Amâncio, L. (1999). Asymmetries in the Perception of Other as a Function of Social Position and Context. Swiss Journal of Psychology, 58, 40-50.

- Cabrera, A.; Flores, T. \& Mata, M. J. (2012). O Feminino Como "Intruso" na Política: uma Análise do Contexto Histórico e da Representação Fotojornalística das Deputadas Portuguesas no Parlamento Paritário e nas Discussões da Lei da Paridade. Media \& Jornalismo, 21, 77-127.

- Cardoso, C. (2009). Padrões e Identidades nas Capas de Newsmagazines: 1999/2009. In Actas Digitais IV Congresso SOPCOM - Sociedade dos Media: Comunicação, Política e História dos Media. Lisboa: ECATI-ULHT, 4331-4343.

- Carter, C. \& Steiner, L. (2004). Mapping the Contested Terrain of Media and Gender Research. In C. Carter \& L. Steiner (ed.), Critical Readings: Media and Gender (pp.11-32). Glasgow: Open University Press.

- Cerqueira, C. (2012). Quando Elas (Não) São Notícia: Mudanças, Persistências e Reconfigurações na Cobertura Jornalística sobre o Dia Internacional da Mulher em Portugal (1975-2007). Originalmente presentado como tesis doctoral, Universidade do Minho.

- Cerqueira, C.; Ribeiro, L. \& Cabecinhas, R. (2009). Mulheres \& Blogosfera: Contributo para o Estudo da Presença Feminina na 'Rede'. Ex aequo, 19, 111-128.

- Croteau, D.; Hoynes, W. \& Milan, S. (2012). Media/Society: Industries, Images and Audiences ( $4^{\mathrm{a}}$ ed.). Los Angeles, London, New Delhi, Singapore, Washington DC: Sage Publications.

- Dolan, K. (2004). Voting for Women: How the Public Evaluates Women Candidates. Boulder, CO: Westview Press.

- Ferin-Cunha, I. (2007). A Televisão das Mulheres: Ensaios Sobre a Recepção. Lisboa: Quimera.

- Gallagher, M. (1981). Unequal Opportunities: the Case of Women and the Media. Paris: UNESCO.

- Gallagher, M. (2001). Gender-Setting: News Agenda for Media Monitoring and Advocacy. Londres: Zed Books.

- Gallagher, M. (2006). Perspetiva Feminista sobre os Média. Ex aequo, 14, 11-34.

- Gallego, J. (2009). Género e Representação Pública: Realidades e Desejos. Media \& Jornalismo - Género, Media e Espaço Público, 8 (15), 43-53.

- Gamson, W.; Croteau, D.; Hoynes, W. \& Sasson, T. (1992). Media Images and the Social Construction of Reality. The Annual Review of Sociology, 18, 373-393.

- Gidengil, E. \& Everitt, J. (2003a). Conventional Coverage/Unconventional Politicians: Gender and Media Coverage of Canadian Leader's Debates, 1993, 1997, 2000. Canadian Journal of Political Science, 36(3), 559577.

- Gidengil, E. \& Everitt, J. (2003b). Talking Tough: Gender and Reported Speech in Campaign News Coverage. Political Communication, 20, 209-232.

- Gill, R. (2007). Gender and the Media. Oxford: Polity Press.

- Glick, P. \& Fiske, S. (1996). The Ambivalent Sexism Inventory. Differentiating Hostile and Benevolent Sexism. Journal of Personality and Social Psychology, 70(3), 491-512.

- Guest, G.; Macqueen, K. \& Namey, E. (2012). Applied Thematic Analysis. Thousand Oaks: Sage Publications. 
- Hall, S. (1980). Encoding/Decoding. In Centre for Contemporary Cultural Studies (ed.), Culture, Media, Language. London: Hutchinson.

- Heldman, C.; Oliver, S. \& Conroy, M. (2009). From Ferraro to Palin: Sexism in Media Coverage of Vice Presidential Candidates. Paper presentado en la American Political Science Association, Toronto, Canadá, 3-6 setiembre.

- Hobbs, R. (2005). The State of Media Literacy Education. The Journal of Communication, 55(4), 865-871.

- Kahn, K. (1994). The Distorted Mirror: Press Coverage of Women Candidates for Statewide Office. Journal of Politics, 56, 154173.

- Kahn, K. \& Goldenberg, E. (1991). Women Candidates in the News: An Examination of Gender Differences in U.S. Senate Campaign Coverage. Public Opinion Quarterly, 55(2), 180-199.

- Kanter, M. (1977). Some Effects of Proportions on Group Life: Skewed Sex Ratios and Responses to Token Women. The American Journal of Sociology, 82, 965-990.

- Kellner, D. (1995). Cultural Studies, Multiculturalism and Media Culture. In G. Dinez \& J. Humez (ed.), Gender, Race and Class in Media: a Text-Reader ( $2^{\mathrm{a}}$ ed.). Thousand Oaks: Sage Publications, 9-20.

- Kim, Y. (2006). How TV Mediates the Husband-Wife Relationship. Feminist Media Studies, 6, 129-143.

- Kim, Y. (2007). An Ethnographer Meets the Mobile Girl. Feminist Media Studies, 7, 204209.

- Kimmel, M. (2000). The Gendered Society. New York: Oxford University Press.

- Lei Orgânica n. 3/2006, de 21 de agosto, Lei da Paridade, Diário da República, 1. ${ }^{a}$ série, N. ${ }^{\circ} 160,5896-5897$.

- Lister, R. (1997). Citizenship: Feminist Perspectives. Basingstoke: Macmillan.
- Lobo, P. \& Cabecinhas, R. (2010). The Negotiation of Meanings in the Evening News: Towards an Understanding of Gender Disadvantages in the Access to the Public Debate. International Communication Gazette, 72(34), 339-358.

- Marktest. (2009). Bareme Imprensa. Accedido el 13 de febrero de 2009 en http://www.marktest.com/wap/.

- Martins, C. (2013). Mulheres, Política e Visibilidade Mediática. As Lideranças de Maria de Lourdes Pintasilgo e de Manuela Ferreira Leite. Originalmente presentado como tesis doctoral, Universidade Nova de Lisboa.

- Mendes, K. \& Carter, C. (2008). Feminist and Gender Media Studies: A Critical Overview. Sociology Compass, 2(6), 1701-1718.

- Mota-Ribeiro, S. (2010). Do Outro Lado do Espelho: Imagens e Discursos de Género nos Anúncios das Revistas Femininas - uma Abordagem Sócio-semiótica Visual Feminista. Originalmente presentado como tesis doctoral, Universidade do Minho.

- Morgan, D. L. (1998). The Focus Group Guidebook. Thousand Oaks, CA: Sage Publications.

- Moscovici, S. (1981). On Social Representations. In J. P. Forgas (ed.), Cognition. London: Academic Press.

- Munday, J. (2006). Identity in Focus: The Use of Focus Groups to Study the Construction of Collective Identity. Sociology, 40/1, 89-105.

- Neuman, W. R.; Just, M. R. \& Crigler, A. N. (1992). Common Knowledge - News and the Construction of Political Meaning. Chicago: University of Chicago Press.

- Nogueira, C. (2006). Os Discursos das Mulheres em Posições de Poder. Cadernos de Psicologia Social do Trabalho, 9(2), 57-72.

- Norris, P. (1997). Women, Media, and Politics. New York: Oxford University Press.

- Ross, K. (1995). Women and the News Agenda: Mediated Reality and Jane Public. 
Discussion Papers in Mass Communication. Leicester, UK: University of Leicester.

- Ross, K. (2002). Women, Politics, Media: Uneasy Relations in Comparative Perspective. Cresskill, NJ: Hampton Press.

- Ross, K. (2003). Women Politicians and Malestream Media: a Game of Two Sides. Center for Advancement of Women in Politics, $1-13$.

- Ross, K. (2004). Women Framed: The Gendered Turn in Mediated Politics. In Karen Ross and Carolyn M. Byerly (ed.), Women and Media: International Perspectives (pp. 60-80). Malden, MA: Blackwell Publications.

- Ross, K. (2010). Gendered Media: Women, Men, and Identity Politics. Lanham, MD: Rowman \& Littlefield Publishers.

- Ross, K. \& Sreberny-Mohammadi, A. (1997). Playing House - Gender, Politics and the News Media in Britain. Media Culture Society, 19(1), 101-109.

- Ross, K.; Evans, E.; Harrison, L.; Shears, M. \& Wadia, K. (2013). The Gender of News and News of Gender: A Study of Sex, Politics, and Press Coverage of the 2010 British General Election. The International Journal of Press/Politics, 18(1), 3-20.

- Santos, M. H. \& Amâncio, L. (2012). Género e Política: Análise sobre as Resistências nos Discursos e nas Práticas Sociais Face à Lei da Paridade. Sociologia, Problemas e Práticas, 68, 79-101.

- Silveirinha, M. J. (1998). O Discurso Feminista e os Estudos dos Media: em Busca da Ligação Necessária. Accedido el 23 de febrero de 2014 en http://www.bocc.ubi.pt/pag/silveirinhamaria-joao-discurso.pdf.

- Silveirinha, M. J. (2004). Os Media e as Mulheres: Horizontes de Representação, de Construção e de Práticas Significantes. In M. J. Silveirinha (org.), As Mulheres e os Media (pp.5-12). Lisboa: Livros Horizonte.
- Silveirinha, M. J. (2006). Introdução. Ex aequo - Representações Mediáticas de Mulheres, 5-9.

- Silverblatt, A. (2001). Media Literacy: Keys to Interpreting Media Messages. Westport, CT: Praeger.

- Sreberny-Mohammadi, A. \& Ross, K. (1996). Women MPs and the Media: Representing the Body Politic. Parliamentary Affairs, 49(1), 103-115.

- Teurlings, J. (2010). Media Literacy and the Challenges of Contemporary Media Culture: On Savvy Viewers and Critical Apathy. European Journal of Cultural Studies, 13(3): 359-373.

- Tuchman, G. (1979). Introduction: the Symbolic Annihilation of Women by Mass Media. In G. Tuchman; K. Daniels \& J. Benét (org.), Hearth and Home: Images of Women in the Mass Media (pp.3-38). Nova Iorque: Oxford University Press.

- United Nations. (1979). Convention on the Elimination of All Forms of Discrimination Against Women. Accedido el 20 de enero de 2014 http: / /www.ohchr.org/en/ProfessionalInte rest/pages/cedaw.aspx.

- Van Dijk, T. (2005). Discurso das Notícias e Ideologias. Porto: Campo das Letras.

- Van Zoonen, L. (1994). Feminist Media Sudies. Newbury Park, CA: Sage Publications.

- Walkerdine, V. (2006). Playing the Game: Young Girls Performing Femininity in Video Game Play. Feminist Media Studies, 6, 519. 537.

- Watkins, C. \& Emerson, R. M. (2000). Feminist Media Criticism and Feminist Media Practices. Annals of the American Academy of Political and Social Science, 571: Feminist Views of the Social Sciences, 151-166.

- Wilkinson, S. (1998). Focus Group in Feminist Research: Power Interaction, and the Co-construction of Meaning. Women's Studies International Forum, 21(1), 111-125. 
- Winship, J. (1987). Inside Women's Magazines. London: Pandora.

- Wolf, N. (1992). O Mito da Beleza: Como as Imagens de Beleza São Usadas Contra as Mulheres. Rio de Janeiro: Rocco.
- Young, I. M. (1990). Justice and the Politics of Difference. Princeton: Princeton University Press.

\section{Fuentes de financiación del estudio}

Realizado en el ámbito del proyecto "O Género em foco: representações sociais nas revistas portuguesas de informação generalista”, con la referencia PTDC/CCI-COM/114182/2009, financiado por el FEDER a través del Programa Operativo de los Factores de Competitividad (COMPETE) y por la Fundación para la Ciencia y la Tecnología (FCT), Portugal.

\section{Forma de Citación}

SANTOS, Anabela; CABECINHAS, Rosa y CERQUEIRA, Carla: Leer, Interpretar y (Re)construir: Percepciones de Jóvenes sobre las Mujeres en la Política. Revista Communication Papers, No 5, páginas 74 a 90. Departamento de Filología y Comunicación de la Universidad de Girona. Recuperado el de de 2 de: http://www.communicationpapers.es 